\title{
EL CONCEPTO \\ DE AUTORIDAD NORMATIVA. \\ EL MODELO DE LAS RAZONES \\ PARA LA ACCIÓN
}

\section{Introducción}

1.1. La expresión «autoridad normativa» es, sin duda, problemática. En primer lugar, porque es utilizada para mencionar nociones diferentes y no es seguro que todas tengan un núcleo significativo común. En segundo lugar, en la medida en que se encuentra asociada con determinadas clases de relaciones sociales, típicamente con relaciones de poder o dominio, se la usa de manera encomiástica o peyorativa para expresar actitudes de aprobación o rechazo. Ello dificulta la posibilidad de un acuerdo en el uso teórico o filosófico de esa expresión. De manera general, se admite que la «autoridad normativa» consiste en la habilidad o capacidad de ciertos individuos. Precisamente, la explicación de la naturaleza de esa habilidad constituye un problema central. Aunque el poder fáctico de imposición es presentado, en todo caso, como un ingrediente conceptual básico, no es habitualmente considerado suficiente para esa explicación. Parece, entonces, que lo que se requiere es dar cuenta, en términos a su vez normativos, del título o derecho de tales individuos para ejercitar el poder. (Friedmann, 1990).

En lo que sigue, me propongo limitar la discusión de esa cuestión al caso de la «autoridad jurídica», entendida como autoridad normativa. Es más, el interrogante apunta a las relaciones entre tres maneras de entender ese concepto y sus respectivas consecuencias para el recurrente problema de la «existencia» de las normas. Cada una de esas maneras, conduce a puntos de vista diferentes acerca de aquel problema central. Como las normas afectan el comportamiento de sus destinatarios, la autoridad normativa es un tipo de autoridad práctica. Lo que quiere decir, a tenor de la terminología propuesta por Joseph Raz, aceptada ampliamente en la discusión actual, que las autoridades normativas tienen que ver con las razones para la acción y no -prima facie- con la razones para la creencia de los individuos 
afectados ( $R a z, 1990)$. De acuerdo a esta propuesta, las nociones de la filosofía práctica, verbigracia, las de «autoridad» y de «norma» tienen que ser construidas en términos del más primitivo concepto de «razones para la acción». Precisamente, me propongo analizar críticamente una de las postuladas derivaciones de esta hipótesis metodológica, a saber, la primacía conceptual de la «autoridad legítima», en tanto autoridad normativa. Primacía que se funda en la idea según la cual los problemas conceptuales no son independientes de los problemas de justificación. En verdad, el punto de controversia consiste en la identificación de toda autoridad normativa con la autoridad moral. En la literatura, se proponen argumentos alternativos en favor de esta asimilación. En este trabajo, la pregunta apunta al alcance del que puede denominarse el «argumento de la coordinación».

1.2. No obstante las divergencias posibles, existen ciertos rasgos estructurales que se pueden asociar con la noción de «autoridad jurídica». Se trata de un concepto relacional en más de un sentido. En primer lugar, el individuo x o el conjunto de individuos al que se califica de autoridad normativa, sólo lo es en relación a otro conjunto de individuos, que puede denominarse la clase $\mathrm{C}$ de los sometidos a $\mathrm{x}$. Es un requisito plausible de adecuación que la clase $\mathrm{C}$ se considere idéntica a la clase de los eventuales destinatarios de las normas formuladas por $\mathrm{x}$. En segundo lugar, los individuos que constituyen la autoridad lo son únicamente en relación a una acción o a una definida clase de acciones que los sometidos pueden realizar. Este carácter estructural determina que tenga que hablarse, más bien, de relaciones de autoridad (Lagerspetz, 1989). El uso no relativizado de «autoridad» supone una autoridad absoluta, resabio de un enfoque teológico según el cual cabe adjudicar a las autoridades empíricas propiedades semejantes a las que se atribuyen a Dios ${ }^{1}$. La índole de esa relación depende de las distintas maneras en que puede entenderse la autoridad normativa. Los «límites» de cada

${ }^{1}$ Raz sostiene que cualquier autoridad política (jurídica) pretende autoridad ilimitada sobre la clase de acciones, aun cuando de hecho, tenga en el tiempo t, límites constitucionales a su competencia porque, en cualquier caso, la constituciónpuede ser modificada. Esta constatación le sirve para argumentar que no es posible justificar la autoridad porque, entre otras cosas, no es posible justificar esa pretensión. Esto es, la autoridad vendría a pretender autoridad absoluta en la dimensión de las acciones. Pero tenga o no semejante pretensión, es claro que cualquier autoridad actual tiene límites actuales, los que podrán reducirse o ampliarse, pero no eliminarse. Lo que puede estar en cuestión es, precisamente, justificar la autoridad actual (confr, $R a z, 1986$, pp. 76 y ss.). 
autoridad están, entonces, determinados por la estructura relacional del concepto.

1.3. Adicionalmente, las relaciones de autoridad tienen una dimensión temporal. Las autoridades comienzan a «existir» en un cierto tiempo t y dejan de existir en otro. En el lapso intermedio, su existencia se manifiesta en una serie de actos autoritarios, esto es, en los actos de creación o formulación de normas. Por consiguiente, salvo el caso atípico de una autoridad para «crear» sólo una norma, las autoridades son relativamente permanentes, y por lo tanto, se refieren a una clase de tales actos ( $R a z, 1978$, p. 20). La cuestión es, entonces, determinar el alcance de expresiones del tipo «x tiene autoridad sobre la clase de individuos $\mathrm{C}$, con respecto a la clase de acciones A en el tiempo $\mathrm{t} 1-\mathrm{t} 2$ ».

\section{Autoridad y normas}

2. 1. El derecho de una cierta sociedad constituye un conjunto de normas que se presenta lingüísticamente como un conjunto de prescripciones. Su existencia implica que el comportamiento de sus miembros se encuentra regulado, esto es, se torna no opcional: con respecto a algunas alternativas de acción, precisamente las previstas en el contenido de las normas, se tiene el deber de actuar o de no actuar ${ }^{2}$. Aunque posibles, tales alternativas están excluidas del control individual, con prescindencia de intenciones, intereses o preferencias subjetivas. La decisión en favor o en contra de su realización es, entonces, impuesta heterónomamente. Ello no significa una determinación causal directa: las normas pueden, de hecho y en todo caso, ser obedecidas o desobedecidas (sobre esta cuestión, Von Wright, 1980, pp. 42-44, 57). Más bien, una manera de expresar esa exclusión, y por consiguiente, el sentido del deber, al menos en lo que se refiere a las normas jurídicas, consiste en indicar que el control motivacional sobre esas determinadas categorías de acciones se produce o pretende producirse indirectamente vía la coacción física no consentida -a la que Nozick denomina «coacción fundamental»- asociada por las propias normas a los actos de desobediencia. Otra perspectiva, menos pesimista, aunque admite la asociación del derecho con la coacción, toma en

${ }^{2}$ Es obvio que las normas no sólo imponen deberes. También conceden facultades o permisos. No obstante, la exclusión de alternativas depende básicamente de las normas de obligación. 
cuenta el punto de vista de los que aceptan el contenido de las normas, y sugiere que son estos los decisivos para comprender la función de su incidencia en la exclusión de alternativas. Tener un deber, es, para los aceptantes, lo mismo que tener una razón para actuar. Las razones para la acción son alteradas por las normas, es más, ellas mismas son tales razones. La amenaza de la coacción sólo cumple una función subsidiaria y, en todo caso, destinada a suministrar una motivación estratégica adicional para asegurar el cumplimiento. Los que cumplen las normas para evitar consecuencias negativas a sus intereses, no reproducen, a tenor de este enfoque, la forma típica en que los comportamientos son regulados normativamente. Esta dicotomía de puntos de vista sobre la naturaleza de las normas que, a partir de Hart, son denominados, «externo» e «interno» respectivamente, puede ser utilizada como hilo conductor, en la medida en que supone planteamientos divergentes para delimitar el problema de la noción de autoridad.

2.2. La suposición de que algunos individuos tienen que ser considerados autoridades normativas conduce a admitir que algunas de las normas son los resultados de actos autoritarios. Según esta manera de hablar, tales actos «crean» las normas, transforman el mundo previo. Esto es, imponen deberes y suministran razones para la acción. Como es claro, tal suposición depende de lo que se quiera decir al afirmar que una norma determinada existe. El término «norma» es también sistemáticamente ambiguo. Si se supone que el dato primario es un conjunto de prescripciones, la pregunta por la existencia apunta a una definida prescripción. Se tienen, al menos, tres respuestas relevantes (Bulygin, 1990, también Raz, 1990b, pp. 80 y ss.): a) una norma existe si la respectiva prescripción pertenece a un sistema de normas; b) una norma existe si la prescripción es vigente o eficaz, esto es, si es generalmente obedecida por sus destinatarios, si existe, de hecho, una práctica establecida de cumplimiento; c) una norma existe si la prescripción tiene fuerza obligatoria, si es vinculante. La existencia en este último caso es, con frecuencia, asimilada a la validez de las normas, aunque «validez» tampoco es un término unívoco. De acuerdo a la clásica versión kelseniana, aceptada explícitamente por Raz, por ejemplo, sólo existen normas válidas en este sentido ( $R a z, 1977)$. Esta primacía significa que la «obligatoriedad» requerida constituye un plus sobre cualquier dato empírico, impuesto por la propia naturaleza de las normas.

Ello se explica por la tentativa de dar cuenta del sentido de la exclusión de la opción individual. Las normas excluyen alternativas 
imponiendo el deber de actuar o de no actuar. Pero últimamente, se sostiene, desde variadas perspectivas, que un genuino deber sólo vendría a existir si el requerimiento expresado en su contenido está finalmente justificado, o lo que es lo mismo, si impone un deber moral (Nino, 1985; Raz, 1984; Finnis, 1988). Si se acepta esta tesis, como así también la paralela equivalencia terminológica entre «validez»y «fuerza vinculante», entonces sólo son válidas, i.e. «existen», las normas morales y no hay distinción que hacer entre derecho y moral, si es que hay que admitir la existencia de normas jurídicas. Las tres nociones de «existencia» son independientes y, por lo tanto, no son recíprocamente excluyentes. Una prescripción puede existir como norma en cualquiera de los sentidos simultáneamente. No obstante, constituye un problema mostrar de qué manera pueden ser coextensivos, más allá de la mera coincidencia.

\section{Nociones de autoridad normativa}

3. 1. Ese problema puede ser discutido en relación a la idea de autoridad normativa. Ello, porque para cualquiera de las condiciones de existencia se tiene una noción correspondiente de «autoridad». Se puede aceptar de manera general, que cada acto de formulación de una norma, se manifiesta en el uso de un lenguaje prescriptivo (u otras formas simbólicas). El primer efecto es que cierta conducta o ciertos tipos de conducta pueden ser calificados como «obligatorios», «prohibidos» o «permitidos», conforme a ese acto lingüístico. Pero la comprobación empírica de que el acto ha tenido lugar, no es suficiente para establecer la existencia de una norma. Un requisito adicional exige que los individuos usuarios del lenguaje puedan ser considerados competentes, en relación a un sistema de normas Sn. Ello significa que, a su vez, Sn contiene normas que autorizan o permiten, bajo ciertas condiciones de procedimiento, la realización de actos de ese tipo, a ciertos individuos identificados por la satisfacción de definidas condiciones. Entonces, tales actos son interpretados como actos de creación o de promulgación de normas, y los individuos en cuestión son, consecuentemente, considerados autoridad normativa, en ambos casos, relativamente al sistema $\mathrm{Sn}$.

El «título» para crear normas equivale aquí a facultamiento y la respectiva autoridad normativa puede denominarse autoridad legal. Sus límites, es decir, la clase de los destinatarios eventuales de las prescripciones, y la clase de actos que le está permitido regular están determinados por las normas de Sn que establecen 
la competencia o facultamiento. Los actos de la autoridad legal son suficientes para establecer la existencia de unas normas en el primer sentido de pertenencia a un sistema normativo y, en verdad, suministran un criterio para reconstruir sistemáticamente un material normativo en términos de actos semejantes: una norma N pertenece a Sn («existe» en Sn) si ha sido promulgada por una autoridad $\mathrm{x}$ de $\mathrm{Sn}$, de acuerdo a una norma de facultamiento N1 de Sn (confr. Kelsen, 1990, capítulo 26). Se trata aquí de una noción de autoridad que depende, meramente, de la descripción de una específica relación entre ciertos actos y el contenido de específicas formulaciones lingüísticas previamente identificadas como el sistema Sn. Esta descripción nada permite inferir acerca del valor moral de las normas de Sn ni de su grado vigencia o eficacia. Pero, en verdad, suministra el primer criterio usual para identificar autoridades jurídicas, para el que el carácter reglado de los actos autoritarios constituye una condición definitoria.

3.2. El paso del segundo sentido de «existencia» de normas, no es, en principio, demasiado problemático. Si lo que interesa es determinar cuáles son las normas efectivamente vigentes en una sociedad determinada, ello se logra imponiendo como condición necesaria la eficacia del sistema Sn al que pertenecen las normas de competencia. Lo que conduce a echar mano a una noción de autoridad explicable en términos de hechos adicionales a los actos de promulgación. Cuando las formulaciones prescriptivas de ciertos individuos son, entonces, obedecidas en forma general por los miembros de esa sociedad, tales individuos pueden calificarse como autoridad efectiva y sus prescripciones son normas «existentes» en el segundo sentido. Por lo pronto, en consecuencia, toda autoridad legal es también autoridad efectiva. Sin embargo, como se ha encargado de mostrar Hart (Hart, 1961), los hechos que constituyen la existencia de una norma en cuanto práctica, son mucho más complejos que la mera reiteración de actos de obediencia. Su descripción tiene que incluir, no sólo la constatación del control de los medios de coerción, sino también y principalmente, las actitudes y disposiciones de comportamiento en que consiste la práctica de aceptación de normas. Ciertos individuos, en condiciones de imponer sus prescripciones, pretenden autoridad, y aceptarlas equivale al reconocimiento de esa autoridad. Este complejo actitudinal recíproco es lo que los convierte en autoridad efectiva. Si, además, se admite la tesis de Hart, según la cual la disposición a actuar conforme a las prescripciones de la autoridad efectiva. Si, además, se admite la tesis de Hart, según la 
cual la disposición a actuar conforme a las prescripciones de la autoridad así reconocida, equivale a la existencia de una regla social que determina los límites de su competencia, entonces también vale que toda autoridad efectiva es también una autoridad legal en relación a esa regla ${ }^{3}$. Pero como es claro, el reconocimiento tampoco suministra un criterio para pronunciarse sobre el valor moral de las normas, y por consiguiente no las convierte en normas válidas.

3.3. Una manera de interpretar ese reconocimiento por parte de los destinatarios de las normas de la pretensión de autoridad de ciertos individuos, es pensar que equivale a una creencia en su legitimidad. Lo que significa concederles lo que E. Garzón Valdés propone denominar legitimación. Los juicios sobre legitimación, aceptación o reconocimiento son empíricos porque se refieren a las efectivas actitudes compartidas en una sociedad con respecto a su sistema jurídico-político (Garzón Valdés, 1987). Aquello en lo que creen proporciona un indicador, según opina Raz, para una genuina elucidación de la noción de autoridad. Creen en que tales individuos constituyen una autoridad legítima. O lo que es lo mismo, creen en la existencia de razones que justifican el ejercicio del poder ( $R a z$, 1983; Raz, 1990c). La pretensión de autoridad que caracteriza a la autoridad efectiva no sería otra cosa que la invocación -aun en última instancia- de un derecho a emitir prescripciones válidas, que no resulta, meramente, de las normas de competencia. Más bien, la pretensión apunta a un derecho moral a ordenar y a ser obedecido y, por consiguiente, los individuos que la constituyen también creen que sus actos autoritarios están justificados moralmente. El título, entonces, tiene carácter moral. Ello significa que toda autoridad normativa se presenta como autoridad moral (Raz, 1984; Soper, 1989; Garzón Valdés, 1990a) y el reconocimiento tiene el sentido de admitirla como autoridad moral. Existe una autoridad legítima cuando semejante creencia está justificada, cuando efectivamente esos individuos son autoridades morales, cuando verdaderamente tienen el derecho a imponer restricciones a los comportamientos de los sometidos. Sólo entonces los actos autoritarios crean

${ }^{3}$ Conf. Hart, 1982, pp. 258-259. Si esa regla se identifica con la regla de reconocimiento, ello equivale a concebirla ahora como una regla de cambio. Esta conclusión es discutible y se asocia con la necesidad de explicar por qué la autoridad «originaria» es también una autoridad, si en todo caso, se precisa normas de competencia para su definición. No voy a abordar en este trabajo este problema. 
normas válidas, auténticas normas que, por lo tanto, deben ser obedecidas.

La primacía conceptual otorgada por Raz y otros a la «autoridad legítima», en el sentido de que comprender la noción de autoridad es lo mismo que mostrar el tipo de argumento que justifica la pretensión de autoridad, apunta a la construcción de un modelo ideal al que deberían adecuarse los comportamientos de los detentadores del poder de imposición ( $R a z, 1990 \mathrm{c}$, pp. 125-126). Los juicios sobre la legitimidad son, por consiguiente, en cualquier caso normativos. Por un lado, sirven para formular requerimientos que las pretendidas autoridades tienen que cumplir. Por el otro, para sustentar exigencias de obediencia dirigidas a los sometidos o destinatarios. Ello, sumado a la tesis de que sólo existen las normas válidas, equivale a afirmar que las autoridades normativas sólo son posibles, de hecho, en la medida en que es posible la satisfacción de ese ideal. La cuestión es, ahora, si se sostiene esta verdadera vuelta de tuerca en el retorno al jusnaturalismo, mostrar de qué manera la misma no conduce a tornar irrelevantes las otras nociones de «autoridad», en tanto criterios eventuales para la identificación de normas jurídicas. La estrategia que voy a examinar consiste en convertir a la «autoridad efectiva» en condición necesaria de la autoridad legítima. Lo que supone que las descripciones de ciertos hechos integran necesariamente, al menos, un argumento de justificación de la autoridad, precisamente, el «argumento de la coordinación».

\section{Puntos de vista sobre la autoridad}

4. 1. Usualmente, el primer paso en favor de la primacía conceptual de la «autoridad legítima» consiste en conceder un privilegio al denominado punto de vista interno en tanto modo de aproximarse a la dimensión de las normas. Ello es claro, en la afirmación de Raz de que se puede percibir lo que es la autoridad «viendo lo que uno reconoce cuando reconoce que una persona tiene autoridad legítima» $(R a z, 1983$, p. 369).

No obstante, es claro que tanto el concepto de «autoridad legal» como el de «autoridad efectiva» pueden ser utilizados por el observador de un sistema jurídico, cuyo interés apunta eventualmente a formular una teoría descriptiva desde el punto de vista externo. Bajo la condición de eficacia del sistema puede constatar que las autoridades legales son también autoridades efectivas en una definida sociedad. Puede, además, comprobar, en un nivel superficial de observación que no sólo las prescripciones de esa 
autoridad son habitualmente obedecidas, sino también que los actos de desobediencia son recurrentemente castigados. Adicionalmente, puede explicar tales comportamientos describiendo el hecho más complejo de que parte de los miembros de la sociedad, y en especial los que detentan el poder coactivo de imposición, aceptan las normas, las cumplen y las hacen cumplir voluntariamente.

El observador formula, entonces, lo que Hart denomina «enunciados externos» sobre el derecho, que no suponen ni implican ningún juicio evaluativo sobre la justificación moral de las autoridades, ni sobre el valor moral de las prescripciones practicadas. Se trata, por lo tanto, del uso de una noción neutral de autoridad, referida meramente a la comprobación de circunstancias empíricas. Comprobación que puede, asimismo, incluir la indicación de que las prácticas de promulgación y de obediencia producen un orden estable de coordinación, que algunos de los miembros -no necesariamente todos- de la sociedad consideran legítimo. De nuevo, ello no requiere abrir juicio sobre la cuestión de saber si esa creencia está o no justificada. Desde esta perspectiva, el alcance de las relaciones de autoridad, o lo que es lo mismo, la pertenencia a la clase $\mathrm{C}$ de sometidos, se determina por el alcance de la capacidad de imposición. De nuevo, como el sistema es, por hipótesis, eficaz, la clase $\mathrm{C}$ coincide con la de los destinatarios eventuales, determinada por las normas de competencia.

Como es notorio, el observador externo puede interesarse también en formular juicios evaluativos sobre el sistema en cuestión, desde la perspectiva de un modelo ideal. Pero se trata, en todo caso, de un paso distinto y adicional que no afecta el carácter descriptivo de formulaciones del tipo « $\mathrm{x}$ es autoridad sobre la clase $\mathrm{C}$ » $\mathrm{O}$ « $\mathrm{N}$ es una norma en el sistema jurídico $\mathrm{S} »$.

4.2. Sin embargo, una teoría de este tipo es considerada insuficiente en la medida en que no reproduce la manera en que se utilizan las normas en el juego interno del sistema. A tenor de una opción actualmente compartida, el punto de vista externo, que pretende reconstruir el positivismo neutral, no sirve para construir una teoría participativa, esto es, una teoría desde el punto de vista de los aceptantes de las normas, quienes en todo caso, se sirven de una noción comprometida de autoridad normativa (Goldsworthy, 1990; Soper, 1989). Según Hart, estos formulan enunciados internos del tipo «la norma N es válida» cuyos contenidos no pueden reducirse a enunciados puramente fácticos. 
Desde el punto de vista interno, las cuestiones normativas son cuestiones prácticas. Tienen que ver con lo que uno debe o no debe hacer, y con las razones para justificar las decisiones. Por consiguiente, también los enunciados que manifiestan el reconocimiento de autoridad están destinados a resolver cuestiones de ese tipo: si x es una autoridad, entonces se debe actuar de acuerdo a las prescripciones de x. Es más, la conclusión normativa está implícita en la propia noción de autoridad, toda vez que no hay autoridad si no existe ese deber. Lo que significa que existe una razón para realizar la conducta prescripta por x, o lo que es lo mismo, que semejante conducta se justifica porque se justifica conducirse conforme a las prescripciones de $\mathrm{x}$. Los enunciados externos no pueden captar esta dimensión. Es por ello que, según Raz, la noción de autoridad legal o autoridad en relación a un sistema $\mathrm{S}$ es insuficiente: el mero hecho de la promulgación de una prescripción por individuos autorizados, no basta para justificar en forma no relativizada que se tenga o no se tenga que realizar una determinada conducta. Hay que admitir, además, que tales individuos constituyen una autoridad legítima. ( $R a z, 1978, R a z, 1990 \mathrm{c}$ ).

4.3. De manera que, entonces, la pretensión de autoridad de $\mathrm{x}$ equivale, desde este punto de vista, a pretender que sus pronunciamientos, esto es, sus prescripciones, constituyan razones perentorias o excluyentes para la acción de sus destinatarios, más allá de la probabilidad del castigo. Se reconoce la autoridad de $\mathrm{x}$, cuando se acepta que sus prescripciones son tales razones. Son razones excluyentes, en el sentido según el cual, al mismo tiempo que requieren una definida conducta, eliminan de la consideración o balance de razones, cualquiera sea su peso, todas o algunas de las razones en contra de la realización de la acción. Se trata de la conocida propuesta de Raz, admitida últimamente por Hart como un modo de explicitar las actitudes normativas que definen la relación de autoridad (Hart, 1982, pp. 253 y ss.).

\section{Autoridad y razones para la acción}

5.1. Pero, ¿qué significa precisamente esa propuesta de las normas como «razones para la acción»? Como es notorio, en la respuesta termina la coincidencia entre Hart y Raz. No es mi intención aquí someter a escrutinio la complicada teoría de Raz sobre el razonamiento práctico. Para la presente discusión, basta con indicar que hay dos maneras de pensar la «existencia» de razones (excluyentes o de otro tipo). En la versión de Hart, que 
puede denominarse subjetivista, la prescripción $\mathrm{P}$ que impone la realización del acto $\mathrm{p}$, es una razón para el individuo i si, y sólo si, a) si i es el destinatario de $\mathrm{P}$, la prescripción es para i una razón suficiente para realizar el acto $\mathrm{p}$. Ello significa que $\mathrm{P}$ excluye subjetivamente las razones para $-\mathrm{p}$, y por consiguiente, que $\mathrm{P}$ determina su comportamiento (confr. Von Wright, 1980, pp. 28 y ss.); b) si i no es el destinatario de P, la prescripción es para i una razón necesaria para la justificación del acto $\mathrm{p}$ de los destinatarios de $\mathrm{P}$, con independencia de las razones que efectivamente lo determinen. Lo que quiere decir que, para i, si la prescripción requiere la realización de p, la omisión de p es, en todo caso, una acción injustificada, cualesquiera sean las razones que los destinatarios invoquen en favor de -p. Una «razón» existe en este sentido, cuando al menos existe un individuo i. En este caso, i es un aceptante de P, la que, entonces, es para él una norma válida. Es admisible pensar que los individuos que pretenden autoridad pertenecen a la clase de los aceptantes de sus propias prescripciones, aun cuando no sean sus destinatarios. Pero, en todo caso, para que exista la relación de autoridad, es necesario que algunos de los destinatarios también pertenezcan a esa clase. Sólo entonces, las prescripciones de la autoridad x constituyen para ellos, normas válidas. Este enfoque es, por cierto, compatible con la constatación de la existencia subjetiva de razones ulteriores para el reconocimiento de x como autoridad normativa, porque no supone la irracionalidad de semejantes actitudes.

5.2. De cualquier modo, lo cierto es que conforme a esta versión subjetivista, los enunciados internos de autoridad no pueden ser enunciados sobre la existencia de razones, porque aquí semejante «existencia» es una cuestión empírica relativa a las creencias y actitudes que pueden ser descriptas en enunciados externos. La condición pragmática de que sólo los aceptantes están en condiciones de formular enunciados del tipo «N es válida» o «x es autoridad», muestra que no son juicios verdaderos o falsos sobre tales creencias. Son en cambio, genuinas recomendaciones normativas sobre lo que los destinatarios deben hacer. Por consiguiente, una teoría desde el punto de vista interno no constituye alternativa al punto de vista externo, simplemente porque los enunciados internos no están destinados a suministrar información ${ }^{4}$.

${ }^{4}$ Esto es así para una concepción no cognoscitiva según la cual las normas, y otras formulaciones normativas, no son verdaderas ni falsas. Pienso que en este punto radica la principal diferencia filosófica entre Hart y Raz, que condiciona sus respectivas versiones del punto de vista interno. 
No obstante, la postulada primacía del punto de vista interno puede tener otro sentido, para la versión subjetivista de las razones para actuar. Puede conducir a la elaboración de un concepto externo de autoridad normativa que es, en todo caso, problemático y no es seguro que haya que adjudicárselo a Hart. Se trata de una noción que asume a la adopción del punto de vista interno por parte de una subclase de los destinatarios, como criterio externo para determinar la clase $\mathrm{C}$ de los sometidos a la autoridad x. De manera que $\mathrm{x}$ tiene autoridad sobre un individuo i si, y sólo, si i reconoce la autoridad de x. O de otra manera, las prescripciones de x sólo son normas para i si las acepta. El reconocimiento del destinatario de las normas es, entonces, para el observador externo, una condición necesaria para afirmar que $\mathrm{x}$ es una autoridad a su respecto. Lo que significa que la clase $\mathrm{C}$ se restringe a los aceptantes, y por lo tanto, su delimitación no depende ahora de las normas de competencia ni del alcance de la capacidad de imposición. Esta manera de establecer el concepto de autoridad elimina de la relación a todos los no aceptantes -obedientes y desobedientes- que, precisamente, son aquellos para los que la exclusión de alternativas significa literalmente imposición y sometimiento. Por consiguiente, no constituye externamente una alternativa plausible, más allá de la mera elección de un lenguaje, porque en cualquier caso habrá que decir que se encuentran sometidos al poder de x. De nuevo, si no se admite esta restricción, la autoridad de x depende de su capacidad de imposición, sin perjuicio de admitir que, de hecho, semejante capacidad es funcional a la existencia de aceptantes.

5.3. La de Raz es, en cambio, una versión objetivista de las razones para actuar (confr. Hart, 1982, pp. 153-161). A pesar de que, a veces (Raz, 1990b, p. 75), utiliza la reconstrucción de una deliberación subjetiva para ilustrar la manera de operar de los tipos de razones que postula -en especial, las razones excluyentes- es claro que sostiene la tesis de que las razones existen como hechos independientes de las creencias, actitudes, disposiciones y comportamientos efectivos de los individuos para quienes, no obstante, son razones para la acción. Porque afirmar que existe una razón para el acto $\mathrm{p}$ significa que $\mathrm{p}$ debe ser realizado y no que alguien está dispuesto a realizar $\mathrm{p}$, o que de hecho, $\mathrm{p}$ se ha realizado. En la terminología de Raz, las razones se aplican a esos individuos y éstos la cumplen cuando realizan el acto requerido aun cuando en el caso no lo hayan determinado subjetivamente ( $R a z, 1990$ b, postscrip, pp. 179 y ss.). Las razones excluyentes, por lo tanto, tampoco son dependientes de la conducta o la 
actitud individual. Como las normas válidas pertenecen a la clase de las razones excluyentes, su existencia es también una cuestión objetiva, aun cuando no existan como prácticas de comportamiento ( $R a z, 1990 b$, p. 76). Inversamente, los que aceptan que ciertas prescripciones son normas válidas, tienen que creer que constituyen razones objetivas para actuar. Pero la validez es distinta que la creencia en la validez: «Solamente las normas válidas son válidas o buenas razones. Las normas practicadas o prescriptas pueden ser llamadas razones sólo en el sentido de la razón (subjetiva) por la que una persona realiza alguna acción... Pero puede ser una mala razón. De otra manera, puede que no sea una razón finalmente, pero algunas personas creen que lo es o intentan que otros la adopten como una razón» (Raz, 1990b, p. 84, también pp. 72-73, agregado y subrayado mío). De manera que, como cuestión objetiva, las normas válidas existen aunque nadie crea en su existencia y a pesar de las creencias erradas de los que practican normas inválidas.

Pero, entonces, la afirmación de que x es una autoridad normativa significa que sus pronunciamientos son objetivamente válidos, que son razones para actuar. Lo que, a su vez, quiere decir que existen razones para aceptar la autoridad de x. Hay que suponer -como cuestión de significado- que cada vez que un aceptante formula enunciados internos de autoridad afirma la existencia de razones excluyentes porque afirmar que $\mathrm{N}$ es válida implica que $\mathrm{N}$ impone un deber, que se debe actuar conforme a N. Sin embargo, sostiene Raz, como el deber supone restricciones al comportamiento de los destinatarios, las razones objetivas para justificar la autoridad, no pueden estar basadas en el autointerés de los individuos que la constituyen. De acuerdo al sentido supuestamente genuino de «validez» y «deber», semejantes razones tienen que ser razones morales, razones que se imponen más allá de los intereses y preferencias individuales ( $R a z, 1990 \mathrm{c}$, p. 126; Raz, 1984). Los que pretenden sinceramente la autoridad y los que la reconocen creen que de hecho tal es la situación objetiva, que sus enunciados internos son verdaderos, que la autoridad es, por consiguiente, legítima.

5.4. La divergencia en el modo de entender el punto de vista interno es la consecuencia de una sustancial alteración del lenguaje de las razones para la acción. Hart no necesita postular que los aceptantes creen en semejantes razones, porque desde su perspectiva los enunciados internos no son enunciados sobre hechos, morales o de cualquier otro tipo. Lo único que se precisa aquí es afirmar que el reconocimiento de la autoridad y la 
aceptación suponen, como condición mínima, el cumplimiento voluntario de las normas, que excluye como eventual razón subjetiva solamente el temor a la sanción. La idea de que, además, implica la creencia en su «corrección» lo puede conducir, inconsistentemente, al otro paradigma, como recientemente lo ha indicado Ernesto Garzón Valdés (Garzón Valdés, $1990 \mathrm{a})^{5}$.

En cambio, para la versión objetivista, la creencia en las razones morales define la condición de aceptante porque los enunciados internos afirman su existencia. Si se admite esta tesis, es coherente sostener que el punto de vista interno es una alternativa cognoscitiva al punto de vista externo: mientras los enunciados externos se refieren a conductas y actitudes subjetivas, los internos versan sobre razones objetivas. Ambos conjuntos de enunciados no pueden ser equivalentes. La primacía conceptual indica que es mejor una noción comprometida de autoridad para comprender lo que significa pretender y reconocer autoridad (Raz, 1986, p. 64). Pero, una vez que se arriba a este punto de la discusión, el resultado no puede ser otro que la perplejidad: ¿en qué sentido semejante comprensión suministra un criterio para identificar a ciertos individuos como la autoridad legítima y por lo tanto, para identificar sus prescripciones como normas válidas? ¿Por qué la dimensión interna se presenta como un punto de vista normativamente privilegiado para establecer cuando existe el deber moral de obediencia? El hecho de que en esa dimensión las prescripciones son asumidas como razones excluyentes para actuar, es notoriamente insuficiente, aun para mostrar la posibilidad de la autoridad legítima. El anarquista que niega esa posibilidad no necesita negar ese hecho. Le basta aducir que esas creencias son sistemáticamente falsas, y, en última instancia, irracionales (confr. Wolff, 1990).

Como respuesta se puede sostener que el reconocimiento de la autoridad es constitutivo de la autoridad legítima. Si ello fuera así, toda autoridad efectiva es ipso facto legítima $\mathrm{y}$, por lo tanto, las correspondientes creencias se autoverifican. Como es notorio, sin embargo, Raz admite que las creencias en las razones objetivas pueden ser falsas. No obstante, los que adoptan el punto

${ }^{5}$ Como en su versión prescriptivista, los enunciados internos no tienen valores de verdad, su formulación por un individuo no está pragmáticamente destinada a expresar una creencia, sino, en todo caso una intención, lo que Kelsen denominaba el «sentido subjetivo». Es claro que esto no quiere decir que el individuo en cuestión carezca de cualquier creencia la que, en todo caso, precisa de otro tipo de lenguaje para expresarse. Por consiguiente, la afirmación de que los aceptantes siempre creen en el valor moral de las normas es una tesis adicional que no se sigue de esta noción de «enunciado interno». 
de vista interno están inhabilitados, en la medida en que se los considera racionales, para determinar su falsedad, porque si creen que $\mathrm{p}$, creen que $\mathrm{p}$ es verdadera y no creen que $\mathrm{p}$ es falsa donde $\mathrm{p}$ es igual a «x debe ser obedecido» (conf. Puga, da Costa, Vernengo, 1990; Garzón Valdés, 1990a; Laporta, 1990). Para establecer que la creencia en la autoridad legítima es falsa hay que comenzar con la duda admitiendo que puede ser falsa, y ello implica, por definición, el abandono del punto de vista interno. De manera que, finalmente, tanto la cuestión de saber si determinados individuos tienen que ser calificados como autoridad efectiva, como la de verificar su eventual legitimidad es un asunto del que pueden ocuparse los que no aceptan las normas, en el sentido fuerte definido por Raz. Es decir, por los que pueden cuestionar la actual existencia de razones objetivas para actuar, porque no creen en la verdad de los correspondientes enunciados.

\section{Autoridad legítima y coordinación}

6.1. Desplazar la cuestión de la legitimidad de la autoridad al punto de vista externo conduce a requerir a aquel que lo adopta, incluyendo al observador, que no se limite a la mera constatación de circunstancias fácticas, sino que, además, en la tarea de identificar normas recurra a un concepto comprometido de autoridad. Ahora, en el sentido de indicar, como cuestión objetiva sobre las razones existentes, cuáles son las prescripciones que los destinatarios deben aceptar, aun cuando de hecho no se las acepte, y cuáles no deben aceptar, no obstante sus actitudes subjetivas. Habida cuenta que las normas genuinas son razones excluyentes, directivas de ese tipo, indican en qué caso deben prescindir de sus preferencias e intereses en tanto determinantes de la acción. Como está en condiciones de establecer la falsedad de las creencias, también puede establecer las condiciones que las tornan verdaderas. En tanto cuestión de teoría acerca de las normas y de la autoridad jurídica, este requerimiento implica que el observador tiene que usar un criterio normativo en su tarea de elucidación conceptual. Tiene, en definitiva, que construir la clase de concepto que Raz denomina normativo-explicatorio ( $R a z$, 1986, p. 63 y ss.). Como es obvio, ello supone que se acepta la discutible ontología de las razones objetivas y la correspondiente función que se asigna al discurso destinado a determinar la existencia de normas. La controversia metateórica puede dirimirse mediante la simple constatación final del uso de conceptos divergentes e irreductibles de «norma» y «autoridad normativa». Pero 
los partidarios de la noción comprometida pueden insistir que su versión, a la vez que reproduce el significado de la pretensión de autoridad, que el positivismo no puede captar, no conduce necesariamente a desechar cualquier apelación a los hechos (Soper, 1989). Basta con sostener que la cuestión de saber si x tiene autoridad no depende exclusivamente de los hechos. En esta dirección se encuentra el tradicional argumento, rescatado bajo sofisticada forma por autores como Raz y Finnis, según el cual, la autoridad se justifica porque constituye un instrumento para resolver problemas de la coordinación necesaria para la obtención del «bien común» o del «interés general».

6.2. Para arribar a ese argumento, es preciso indicar que se tienen dos estrategias generales posibles para fundamentar la legitimidad del Estado, de un sistema jurídico político en particular o de las instituciones que conforman sus autoridades normativas. La primera, recurre a razones relativas al proceso de su constitución, a los hechos que originan una determinada autoridad. En este caso, la legitimidad es una propiedad emergente de ese proceso, de acuerdo a la terminología propuesta por D. Schmidtz (Schmidtz, 1990). Razones que tienen que ver con la forma en que ciertos individuos adquieren el monopolio de la coacción son de este tipo. Si son elegidos por la mayoría de los sometidos o eventuales destinatarios o si de alguna manera se apela a su consentimiento, la autoridad normativa adquiere legitimidad democrática. De la misma manera, sus prescripciones pueden considerarse justificadas si resultan de un procedimiento democrático de promulgación. En cualquier caso, la justificación es, a su vez, relativa a las razones para avalar la democracia como forma general de gobierno, las que por cierto, imponen límites que definen el proceso admisible. No es mi propósito discutir aquí alternativas de justificación emergente. Pero es claro que la satisfacción de un procedimiento de elección -cualquiera que sea- no puede asegurar ningún resultado, aunque tales límites puedan eliminar algunos resultados, en tanto restricciones a la competencia. En especial, no puede asegurar, en todo caso, la calidad moral de las normas emitidas por la autoridad así legitimada. Por consiguiente, la estrategia en cuestión no habilita ningún argumento para admitir que la autoridad legítima tenga que considerarse una autoridad moral, esto es, una autoridad con derecho moral para emitir normas que sus destinatarios deben moralmente obedecer, por el solo hecho de que son el resultado de actos autoritarios. Lo que, obviamente, no descarta lo que también el anarquista está dispuesto a admitir: que pueden existir, circunstancialmente, otras razones para realizar 
los actos prescriptos (Wolff, 1990, p. 29-30). Identificar como «normas» las prescripciones de la autoridad legítima no es lo mismo, en consecuencia, que reconocer su validez. La insistencia de que, de cualquier modo, es una autoridad moral, obliga a postular como único indicador para determinar el valor moral de las normas, el hecho de su promulgación por semejante autoridad, con total prescindencia de su contenido. Es decir, de la naturaleza de los actos que las prescripciones ordenan practicar. En tal caso, conceder «legitimidad» vendría a ser lo mismo que otorgar competencia formal sobre asuntos morales. Pero esta tesis no se sigue solamente de un determinado argumento de justificación emergente de la autoridad normativa, ni tampoco de la estrategia global. Por consiguiente, puede ser consistentemente rechazado. Se trata, más bien, de un criterio formal e independiente para establecer la validez de las normas, para el cual el origen de la autoridad es indiferente. Ello porque, para cualquier supuesto de su constitución, el criterio indica que el contenido de las normas sigue siendo irrelevante.

6.3. Tanto Raz como Finnis rechazan los argumentos que apuntan a la justificación emergente, por considerarlos insuficientes, con razón, para asegurar la autoridad moral de la autoridad legítima. En especial, no admiten el clásico argumento del consentimiento de los afectados. (Raz, 1986, pp. 80 y ss. Raz, 1990a; Finnis, 1988, pp. 248-252). En principio recurren a la otra estrategia que, también de acuerdo con Schmidtz, puede denominarse teleológica. Aquí, se propone argumentos para justificar la autoridad normativa (o el Estado o cualquiera de sus instituciones) en términos del logro de propósitos o resultados considerados deseables. Para ello, la existencia de una autoridad es considerada condición necesaria para la obtención de un resultado colectivo valioso $\mathrm{R}$. Si R se define por el cumplimiento de las razones morales objetivas que los destinatarios de las normas deben cumplir, ello presupone que los actos de obediencia a la autoridad $\mathrm{x}$ aseguran semejante resultado. Por lo tanto, se precisa aquí un criterio moral, independiente de los actos de promulgación, para saber cuáles son las prescripciones que constituyen normas válidas, esto es las que se deben obedecer. Porque es ese criterio - sin el cual no puede definirse el resultado- el que habilita la justificación teleológica de las autoridades normativas.

Pero en este caso, la prescripción $\mathrm{P}$ que ordena $\mathrm{p}$ no constituye una razón excluyente para realizar $\mathrm{p}$, a menos que exista previamente una razón moral para realizar $\mathrm{p}$, o lo que es lo mismo, que se deba objetivamente realizar p. Sólo entonces, las prescripciones 
se transforman en normas jurídicas válidas, por su concordancia con un deber moral. Consecuentemente, para Raz, las normas jurídicas son razones dependientes para actuar, y esa dependencia es constitutiva de su validez. Las autoridades normativas tienen el deber de promulgar normas válidas. Si cumplen ese deber, entonces son autoridades legítimas, en relación al resultado considerado deseable (Raz, 1990c, pp. 122-129; Raz, 1986, pp. 42 у ss.). Esto es, solo y en la medida en que sus normas son razones dependientes.

No voy aquí a discutir esta línea argumental que, claramente conduce a concebir a la «autoridad normativa» como una especie de autoridad teórica sobre asuntos morales que ejerce una suerte de paternalismo solamente sobre quienes son, para utilizar los términos de Ernesto Garzón Valdés, incompetentes morales (Garzón Valdés, 1990b, confr. para Raz, también Farrell, 1991). Es preciso, no obstante, indicar que en este caso el juicio de legitimidad es siempre ex post facto porque depende de que efectivamente se haya obtenido el resultado valioso. De este hecho nada se puede inferir acerca de lo que deben moralmente hacer en el futuro los destinatarios de las normas después del tiempo $t$ en el que se formula ese juicio sobre el pasado. Sin embargo, Raz recurre, sorprendentemente, a otro argumento -formalmente independiente y hasta incompatible con el otro- para diseñar el concepto de autoridad legítima, que lo conduce al criterio formal para justificar la validez de las normas. Ello explica que explícitamente afirme que sólo son razones válidas para actuar, las normas promulgadas por la autoridad legítima ( $R a z, 1986$, p. 46). La promulgación es, entonces, un criterio de validez. Para mantener la consistencia con la tesis según la cual son razones dependientes, ello tiene que entenderse en el sentido de que la norma $\mathrm{N}$ que ordena $\mathrm{p}$ es una razón necesaria pero no suficiente para realizar p. ¿Pero, por qué hay que admitir que es una razón necesaria? La respuesta es que hay casos centrales en los que la autoridad normativa es realmente una autoridad práctica y no teórica. Lo que significa que antes de la promulgación de $\mathrm{N}$ no existe una razón necesaria para realizar $\mathrm{p}$, porque otras acciones son igualmente suficientes para satisfacer un objetivo moralmente valioso (Raz, 1990c, 126-128).

6.4. Esta constatación es la que permite el paso para asociar los tres conceptos de autoridad. A tenor de Raz, las normas jurídicas son sistemáticamente válidas, porque una condición necesaria de validez es su pertenencia a un sistema Sn. Lo que, al menos, quiere decir que su promulgación por una autoridad legal conforme a Sn es parte de la razón para realizar el acto prescripto. 
(Raz, 1990, pp. 127-128; Raz, Validity). Ello podría entenderse en el sentido de que una vez constatada la legitimidad del sistema -esto es, la legitimidad de sus autoridades-, entonces las normas son sistemáticamente válidas. Por tanto, antes de determinar que la autoridad legal x debe ser obedecida, habría que saber si x es legítima. No obstante, esta afirmación ya es, prima facie, incompatible con el otro argumento de la dependencia de las normas. Porque si la validez depende del contenido no pueden ser sistemáticamente válidas (conf. Galsworthy, 1991, pp. 460-462). Este paso de Raz indica, entonces, que no hay cuestión sobre el contenido de una norma, a menos que antes se establezca su pertenencia a un sistema. Sin embargo, de nuevo, para que las normas de la autoridad legal deban ser obedecidas, es preciso que el sistema sea eficaz; esto es, los sistemas sólo son «sistemáticamente válidos» si existen como prácticas sociales (Raz, 1990b, p. 128). De manera que toda autoridad legítima es también una autoridad efectiva y el hecho de la obediencia habitual es un paso necesario en su justificación. Por lo tanto, la legalidad de los actos autoritarios de ciertos individuos, conforme a las normas de un sistema eficaz, es un requisito de identificación de la autoridad legítima, y no a la inversa (en el mismo sentido, Laporta, 1990).

Ello se sustenta en el hecho de que solamente una autoridad efectiva puede resolver problemas de convivencia. La resolución de estos problemas constituye la razón principal para su justificación (Raz, 1990a; Raz, 1986, p. 76; Finnis, 1988, pp. 231-233, 249; Lagerpetz, 1989, pp. 65 y ss.). En consecuencia, la autoridad no se justifica teleológicamente, ahora, por lo que hace, sino por lo que puede hacer. Los problemas de convivencia pueden ser pensados como conjuntos de problemas de coordinación PC. Pero se tienen dos versiones de problemas PC. En primer lugar, existe un problema PC subjetivo para un conjunto de individuos I, si todos tienen una razón subjetiva para lograr un resultado $\mathrm{R}$ que depende de las acciones conjuntas de los individuos de I y: a) existen estrategias alternativas de acción conjunta, cada una de las cuales es suficiente para obtener R; b) $\mathrm{R}$ se logra si, y sólo si, todos coinciden en elegir una determinada estrategia. Por lo tanto, para cada individuo, es preciso saber cuál será la acción que los otros van a elegir. Los actos autoritarios resuelven el PC, si ordenan adoptar un tipo de acción que todos deben seguir y todos obedecen. El clásico ejemplo de la dirección del tránsito vehicular es un problema subjetivo en este sentido.

Existen varios argumentos -que no serán revisados aquí- para mostrar que la autoridad no es necesaria para resolver problemas subjetivos de coordinación (confr. Green, 1985), o 
para sostener que actuar conforme a las prescripciones no implica reconocer la «autoridad» de los individuos que las emiten (Regan, 1989, pp. 1027-1029). Por ello, consecuentemente, Raz indica que lo que interesa son los PC que pueden denominarse objetivos, esto es aquellos en los que el resultado deseable $\mathrm{R}$ no depende de las razones subjetivas, porque no depende de las preferencias e intereses de aquellos cuya acción es necesaria para R. Ello sucede cuando R se identifica con el «bien común»o el «interés general», para cuyo logro todos tienen una razón objetiva para actuar (Raz, 1990, pp. 6-11; Finnis, 1988, p. 232), con independencia de sus evaluaciones subjetivas. La autoridad normativa, es decir, jurídica, cumple entonces dos funciones: a) define el resultado deseable; b) impone autoritariamente el curso de acción considerado causalmente necesario. De otra manera, sin el esfuerzo coordinado, algunos bienes deseables se pierden o se logran imponiendo cargas excesivas sobre los que cooperan voluntariamente. En consecuencia, solamente la autoridad efectiva, la que es habitualmente obedecida, puede resolver problemas objetivos de coordinación, y sus prescripciones son condiciones necesarias para elegir los actos que deben ser realizados.

6.5. Pero como cuestión empírica no puede descartarse que las normas son, en primer lugar, una herramienta para obtener los estados de cosas que satisfacen los intereses de los individuos que constituyen una autoridad normativa, o para resolver conflictos en beneficio de una clase, o más débilmente, para lo que consideran resultados deseables (Von Wright, 1980, p. 44; Soper, 1989, pp. 222 y ss.). De manera que, para que las normas tengan que considerarse razones suficientes para actuar es preciso saber si se justifica el resultado propuesto en cada problema objetivo de coordinación. Consecuentemente, si sólo deben obedecer si x actúa legítimamente, esto es, si el resultado es objetivamente valioso, entonces los individuos sometidos tienen una razón para la crítica permanente, y no deben asumir las normas como razones excluyentes para actuar.

Pero ello implica, paradójicamente, no reconocer a $\mathrm{x}$ autoridad alguna, porque admitir que $\mathrm{x}$ tiene autoridad es «considerar sus órdenes... como instrucciones autoritarias, y en consecuencia, como razones excluyentes» (Raz, 1990b, p. 63; Finnis, 1988, 233-234). Sin embargo, los resultados colectivos valiosos sólo son posibles si existe una autoridad efectiva que asegure la coordinación, esto es, «si los individuos concernidos [los destinatarios de las normas], difieren su propio juicio y no actúan sobre el balance de razones, sino sobre las instrucciones de la autoridad» $(\operatorname{Raz}$, 
1990b, p. 64). De manera que la legitimidad no puede consistir en el acierto moral en la forma de resolver PC objetivos, sino en la razón ulterior para aceptar que existan autoridades efectivas: a saber, que sólo ellas pueden resolver tales problemas, porque sólo ellas pueden resolver cualquier problema de coordinación.

El razonamiento puede reconstruirse así: si todos tienen una razón objetiva para lograr resultados valiosos y la existencia de una autoridad normativa efectiva es necesaria, todos tienen una razón para que exista una autoridad normativa efectiva. Pero para que exista una autoridad semejante es necesario que sus normas se asuman como razones excluyentes, de manera que todos tienen una razón para asumir las normas como razones excluyentes. Finalmente, cuando una acción (o clase de acciones) es admitida como necesaria para el logro $\mathrm{R}$ y se debe lograr $\mathrm{R}$, entonces $\mathrm{R}$ es una razón suficiente para la acción ${ }^{6}$. Lo que significa que si los actos de obediencia son medios necesarios para el resultado final, para cualquier clase de actos $\mathrm{p}$, la norma que ordena p suministra una razón objetiva suficiente y no meramente necesaria para p. De manera que todos deben obedecer a la autoridad efectiva, si tienen razones para el resultado valioso $\mathrm{R}$, con independencia del contenido de las normas. Por consiguiente, la promulgación de $\mathrm{N}$ por la autoridad legal de un sistema efectivo, tendría que ser considerada suficiente para la validez de N. Esto es, toda autoridad efectiva es, prima facie, legítima si por ello se entiende que sus normas deben ser obedecidas. La clase $\mathrm{C}$ se determina nuevamente por la capacidad de imposición.

6.6. Se trata, por cierto, de una conclusión que el propio Raz estaría dispuesto a negar, toda vez que explícitamente niega ahora que existe una obligación general de obedecer al derecho ( $R a z, 1986$, pp. 99 y ss.). No obstante, hay que indicar que: a) su rechazo a la obligación general, se sustenta en la otra estrategia de justificación que conduce a definir la autoridad normativa como autoridad teórica en asuntos morales; b) la obligación general de obediencia aparece, en cambio, como una consecuencia directa, de la hipótesis que concibe a la autoridad como instrumento necesario para la resolución de problemas PC, en conjunción con la tesis según la cual no hay autoridad si sus normas no son admitidas como razones excluyentes; c) esta consecuencia no equivale a afirmar que se trata de una obligación absoluta. Basta

${ }^{6}$ Confr. Von Wrigth, 1980, pp. 28 y ss. Von Wrigth se refiere al resultado intencionalmente «querido» por el agente. Pero lo mismo vale para el resultado que el agente «debe» querer. 
admitir que los deberes morales son, en cualquier caso, prima facie. En especial, ello significa que la razón objetiva para obtener resultados colectivos deseables puede ser superada, en un caso particular, por otras razones. Por lo tanto, se tienen dos versiones de la autoridad jurídica como autoridad moral, que son recíprocamente inconsistentes.

Lo que explica que Raz mantenga que las normas deben ser obedecidas, es decir, que son razones objetivas excluyentes a pesar de los desaciertos cometidos por los individuos que constituyen la autoridad. Por lo tanto, existen normas que son válidas, no obstante que su contenido pueda ser calificado de inmoral, según cualquier otro criterio (Raz, 1990c, pp. 135-136; Raz, 1986, p. 78-79). Son válidas porque pertenecen a un sistema legítimo. Pero cualquier sistema eficaz instaura autoridades legítimas si la legitimidad se funda en la capacidad técnica para resolver problemas de coordinación. En cambio, en esta misma dirección, Finnis sostiene coherentemente que el hecho de que la autoridad efectiva será probablemente obedecida, tiene consecuencias normativas para lo que los afectados deben hacer. Esas consecuencias se derivan del principio normativo según el cual la «autoridad es un bien (porque es requerida para la realización del bien común) cuando ese principio se asume en conjunción con el hecho de que una persona en particular, un cuerpo o configuración de personas, para una determinada comunidad y en un tiempo definido, pueden hacer lo que la autoridad tiene que hacer (es decir, asegurar y promover el bien común)» (Finnis, 1988, p. 246, subrayados míos). Lo que quiere decir que esta conjunción es una razón suficiente, aunque sea presuntivamente [prima facie], para justificar la pretensión y el reconocimiento de la autoridad (Finnis, 1988, p. 246). Como la manera en que las autoridades efectivas adquieren el monopolio del poder y comienzan a ejercitar el dominio es aquí irrelevante, ello le permite a Finnis concluir que los habitantes de un país ocupado militarmente, con independencia de la moralidad o la inmoralidad de la ocupación, deben obedecer a la autoridad militar efectiva, o que después de todo, la conquista constituye un título bien fundado o razonable para justificar el dominio de la corona británica en sus colonias (Finnis, 1988, nota al párrafo IX. 4 p. 258; nota a pie n. ${ }^{\circ} 15$, p. 252).

Como criterio externo para evaluar sistemas normativos efectivos, no existe razón para aceptar los argumentos morales de Finnis, que personalmente considero inadmisibles. Pero la cuestión importante es que la vía elegida para construir un concepto comprometido de autoridad normativa conduce irremediablemente a que, externamente, haya que postular que las autoridades efec- 
tivas, como derivación de ese «compromiso» aparentemente conceptual, deben ser objetivamente obedecidas. Tesis que, a pesar de su venerable tradición, no es otra cosa que un genuino postulado ideológico acerca del derecho y del poder. El positivista cuyo propósito es el conocimiento no necesita adoptar, por definición, tan fuerte compromiso. Sus conceptos de «norma»y «autoridad» sólo suministran criterios para reconstruir hechos, los que no clausuran la posible e independiente discusión sobre su evaluación moral. Puede ser, entonces, que también para esta controversia, el punto de partida neutral constituya todavía la mejor alternativa.

\section{BIBLIOGRAFÍA}

Bulygin, Eugenio (1990): «An Antinomy in Kelsen's Pure Theory of Law», en Ratio Juris, vol. 3, n. ${ }^{\text {o }}$ $1,1990$.

Farrell, Martin(1991): «Autonomy and Paternalism: the Political Philosophy of Joseph Raz», en Ratio Juris, vol. 4, n. ${ }^{\circ}$ 1, march 1991.

Finnis, John (1988): Natural Law and Natural Rights, Oxford University Press, New York, 1988.

Friedman, R. B. (1990): «On the Concept of Authority in Political Philosophy», ahora incluido en Joseph Raz (ed.) Authority, Basil Blackwell, Oxford, 1990.

Garzón Valdés, Ernesto (1987): El Concepto de Estabilidad de los Sistemas Políticos, Centro de Estudios Constitucionales, Madrid, 1987.

Garzón Valdés, Ernesto (1990a): «Algo más acerca de la Relación entre Derecho y Moral», en Doxa, n. ${ }^{\circ} 8,1990$.

Garzón Valdés, Ernesto (1990b): «On Justifying Legal Paternalism», en Ratio Juris, vol. 3, n. ${ }^{\circ} 2$, 1990.

Go1dsworthy, Jeffrey D. (1990): «The Self-Destruction of Legal Positivism», en Oxford Journal of Legal Studies, vol. 10, n. 41990.

Green, Leslei (1985): «Authority and Convention», en The Philosophical Quarterly, vol. 35, n. ${ }^{\circ}$ 141, 1985.

Hart, Herbert (1961): The Concept of Law, Oxford University Press, Oxford, 1961.

Hart, Herbert (1982): Essays on Bentham, Oxford University Press, New York, 1991.

Kelsen, Hans (1991): General Theory of Norms, Oxford University Press, New York, 1991.

Lagerspetz, Eerik (1989): A Conventionalist Theory of Institutions, Acta Philosophica Fennica, Helsinki, 1989.

Laporta, Francisco (1990): «Derecho y Moral: Vindicación del Observador Crítico y Aporía del Iusnaturalista», en Doxa, n. ${ }^{\circ}$ 8, 1990.

Nino, Carlos (1985): La Validez del Derecho, Astrea, Buenos Aires, 1985. 
Puga, Leila; da Costa Newton; Vernengo, Roberto (1990): «Derecho, Moral y Preferencias Valorativas», en Theoria, año $\mathrm{V}, \mathrm{n}^{\circ}{ }^{\mathrm{o}} 12-13,1990$.

Raz, Joseph (1977): «Legal Validity», en Archiv für Rechts und Sozial Philosopie, LXIII/3, 1977.

Raz, Joseph (1978): «On Legitimate Authority», en Richard Bronaugh (ed.): Philosophical Law, Greenwood Press, Wesport, 1978.

Raz, Joseph (1983): «Autoridad y Consentimiento», en E. Bulygin; M. Farrell; C. Nino y E. Rabossi (eds.) El Lenguaje del Derecho. Homenaje a Genaro Carrió, Abeledo, Buenos Aires, 1983.

Raz, Joseph (1984): «Hart on Moral Rights and Legal Duties», en Oxford Journal of Legal Studies, vol. 4, n. $^{\circ} 1,1984$.

Raz, Joseph (1986): The Morality of Freedom, Oxford University Press, New York, 1986.

Raz, Joseph (1990a): «Introduction», en Joseph Raz (ed.) Authority; Basil Blackwell, Oxford, 1990.

Raz, Joseph (1990b): Practical Reasons and Norms (2. ${ }^{a}$ edic.), Princeton University Press, Princeton, 1990.

Raz, Joseph (1990c): «Authority and Justification», ahora incluido en Joseph Raz (ed.): Authority, Basil BlackweIl, Oxford, 1990.

Regan, Donald (1989): «Authority and Value: Reflection on Raz's Morality of Freedom», en Southern California Law Review, vol. 62, 1989.

Schmidtz, David (1990): «Justifyng the State», en Ethics, 101, october, 1990.

Soper, Philip (1989): «Legal Theory and the Claim of Authority», en Philosophy \& Public Affairs, vol. 18, n. $^{\circ} 3,1989$.

Von Wright, Georg H. (1980): Freedom and Determination, Acta Philosophica Fennica, Amsterdam, 1980.

Wolff, Robert P. (1990): «The Conflict between Authority and Autonomy», ahora incluido en Joseph Raz (ed.) Authority, Basil Blackwell, Oxford, 1990. 